\title{
COVID-19 and cancer patients
}

\section{Dante Luiz Escuissato ${ }^{1}$}

Coronavirus disease 2019 (COVID-19) started in the city of Wuhan, China, and quickly spread around the world, becoming a pandemic. It continues to be a serious public health problem.

On computed tomography (CT), pulmonary infection with severe acute respiratory syndrome coronavirus 2 (SARS-CoV-2) is characterized by ground-glass opacities and consolidations, with a bilateral distribution. Cavitary lung lesions, small pulmonary nodules, and pleural effusion are atypical; when any of those are identified, other causes should be investigated ${ }^{(\mathbf{1 , 2})}$.

The sensitivity and specificity of chest CT vary widely (60$98 \%$ and $25-53 \%$, respectively). The positive predictive value (PPV) and negative predictive value (NPV) are estimated at $92 \%$ and $42 \%$, respectively. Due to the relatively low NPV, the Brazilian College of Radiology and Diagnostic Imaging, as well as several international entities, recommend that chest CT not be used in the screening for COVID-19. However, in a clinical context, its use is justified when diagnostic resources such as reverse-transcriptase polymerase chain reaction and serological tests are not available $\mathrm{e}^{(\mathbf{3 , 4})}$.

More than 18 million new cases of cancer appear each year worldwide. Because of the coexistence of chronic diseases and poor general health status, together with the immunosuppression caused by the neoplasia and the treatment regimens, individuals with cancer are more susceptible to infection. As a consequence, cancer patients infected with SARS-CoV-2, especially those with hematologic malignancies, lung cancer, or advanced metastatic disease, may have a worse evolution and prognosis when compared with other populations of SARS-CoV2-infected individuals ${ }^{(5)}$.

Zhang et al. ${ }^{(6)}$ observed that the chest CT abnormalities seen in cancer patients with COVID-19 pneumonia were similar to those seen in the general population of patients with pneumonia. The findings included ground-glass opacities (in

1. Associate Professor of Radiology at Universidade Federal do Paraná (UFPR), Curitiba, PR, Brazil. Email: dante.escuissato@gmail.com.

https://orcid.org/0000-0002-8978-4897.
75.0\%), multifocal consolidations (in 46.3\%), and bilateral lesions (in 78.6\%). The authors suggested that the worse prognosis in some cancer patients may be related to delayed hospitalization.

In this issue of Radiologia Brasileira, Barbosa et al. ${ }^{(7)}$ presented a study conducted at a cancer center in Brazil where two different approaches were taken to determine whether chest CT findings should be considered positive for COVID-19. The authors observed that, in scenario 1 (in which positivity was defined on the basis of typical chest CT findings alone), sensitivity was lower, whereas specificity and NPV were higher, than what is found in the medical literature. In scenario 2 (in which positivity was defined on the basis of typical and indeterminate chest CT findings), the accuracy was similar to that reported in other studies. In both scenarios, the NPV was higher than that reported elsewhere. These differences reflect the current state of knowledge of this virus, which has various clinical and epidemiological characteristics, raising new questions and calling for ongoing review of concepts.

\section{REFERENCES}

1. Ye Z, Zhang $Y$, Wang $Y$, et al. Chest CT manifestations of new coronavirus disease 2019 (COVID-19): a pictorial review. Eur Radiol. 2020 Mar 19;PMID:32193638. [Epub ahead of print].

2. Zhao W, Zhong Z, Xie X, et al. Relation between chest CT findings and clinical conditions of coronavirus disease (COVID-19) pneumonia: a multicenter study. AJR Am J Roentgenol. 2020;214:1072-7.

3. Colégio Brasileiro de Radiologia e Diagnóstico por Imagem. Recomendações de uso de métodos de imagem para pacientes suspeitos de infecção pelo COVID-19. Versão 3. [cited 2020 Jun 23]. Available from: https://cbr. org.br/wp-content/uploads/2020/03/CBR_Recomendações-de-uso-de-métodos-de-imagem.pdf.

4. Simpson S, Kay FU, Abbara S, et al. Radiological Society of North America expert consensus statement on reporting chest CT findings related to COVID-19. Endorsed by the Society of Thoracic Radiology, the American College of Radiology, and RSNA - Secondary publication. J Thorac Imaging. 2020;35:219-27.

5. Dai M, Liu D, Liu M, et al. Patients with cancer appear more vulnerable to SARS-CoV-2: a multicenter study during the COVID-19 outbreak. Cancer Discov. 2020;10:783-91.

6. Zhang L, Zhu F, Xie L, et al. Clinical characteristics of COVID-19-infected cancer patients: a retrospective case study in three hospitals within Wuhan, China. Ann Oncol. 2020;31:894-901.

7. Barbosa PNVP, Bitencourt AGV, Miranda GD, et al. Chest CT in the diagnosis of SARS-CoV-2 infection: initial experience in a cancer center. Radiol Bras 2020;53:211-5. 